\title{
Risk factors in patients with AFB smear- positive sputum who receive inappropriate antituberculous treatment
}

This article was published in the following Dove Press journal:

Drug Design, Development and Therapy

30 January 2013

Number of times this article has been viewed

\author{
Cheng-Yu Changl,* \\ Jen-Yee Hong ${ }^{2, *}$ \\ Mei-Kang Yuan ${ }^{3,4}$ \\ Shu-Ju Chang ${ }^{5}$ \\ Yuan-Ming Lee ${ }^{6}$ \\ Shih-Chieh Chang ${ }^{2,4}$ \\ Li-Cho Hsu ${ }^{2, *}$ \\ Shin-Lung Cheng' \\ 'Division of Chest Medicine, \\ Department of Internal Medicine, Far \\ Eastern Memorial Hospital, Taipei, \\ Taiwan; ${ }^{2}$ Department of Internal \\ Medicine, ${ }^{3}$ Department of Radiology, \\ ${ }^{4}$ Faculty of Medicine, School \\ of Medicine, National Yang-Ming \\ University, Taipei, Taiwan; ${ }^{5}$ Department \\ of Industrial Management and \\ Enterprise Information, Aletheia \\ University, Taipei, Taiwan; ${ }^{6}$ Department \\ of Laboratory Medicine, National \\ Yang-Ming University Hospital, Yilan, \\ Taiwan \\ *These authors contributed equally to \\ this work
}

Background: Acid-fast bacilli (AFB) smear-positive sputum is usually an initial clue in the diagnosis of pulmonary tuberculosis (TB); however, the test is not disease-specific. Nontuberculous mycobacterium-related colonization or lung disease often has AFB smearpositive sputum results, and physicians may prescribe unnecessary antituberculous drugs for these patients. The aim of this study was to analyze the clinical characteristics of patients with AFB smear-positive sputum who received unnecessary anti-TB treatment.

Methods and patients: From January 2008 to July 2011, we retrospectively enrolled 97 patients with AFB smear-positive sputum who did not have pulmonary TB according to mycobacterial cultures and clinical judgment. We analyzed the clinical and radiographic features of the patients who received inappropriate and unnecessary anti-TB treatment. Preliminary analyses of chisquare and Fisher's exact tests were applied to determine factors unlikely to be associated with the independent variables. The relationship between independent covariates was then analyzed using multivariate logistic regression.

Results: Of the 97 enrolled patients, 25 (25.8\%) were diagnosed with pulmonary TB and prescribed anti-TB drugs (mostly a combination of isoniazid, rifampicin, ethambutol, and pyrazinamide). The other $72(74.2 \%)$ patients were not initially diagnosed with pulmonary TB and were classified as the control group. Compared to the control group, the patients who received inappropriate anti-TB treatment had more chronic cough as presentation symptom and heavy AFB Ziehl-Neelsen staining in sputum ( $>10 / 100$ fields, grading $2+$ to $4+)$. There were no significant differences in the radiographic analysis between the two groups.

Conclusion: Among the patients with AFB smear-positive sputum that did not have pulmonary $\mathrm{TB}$, chronic cough and heavy AFB staining (2+ to 4+) were risk factors for the inappropriate administration of unnecessary anti-TB treatment.

Keywords: AFB smear-positive sputum, Mycobacterium tuberculosis, antituberculous treatment

\section{Introduction}

Pulmonary tuberculosis (TB) remains an important public health problem with an estimated 9.27 million new cases worldwide in $2007 .{ }^{1}$ According to the current treatment guidelines, isolation of Mycobacterium tuberculosis from a sputum culture is still recommended to confirm the diagnosis of pulmonary TB. ${ }^{2}$ However, the decision to initiate TB treatment should be based on epidemiology, clinical/radiographic findings, and the results of acid-fast bacilli (AFB)-stained sputum. In endemic areas, it is not uncommon to administer anti-TB treatment empirically pending culture results, given the clinical features suggesting pulmonary TB. Although this strategy aims at better disease transmission control, it comes with a price: it is possible to inappropriately treat patients
Correspondence: Shih-Chieh Chang Department of Internal Medicine, National Yang-Ming University Hospital, No 152, Xin-Min Road, Yilan City 260, Taiwan

Tel +88639325192 ext 3652

Fax +88 639365432

Email dtsurga9@yahoo.com.tw 
without pulmonary TB with anti-TB drugs, leading to adverse effects and unnecessary costs. In addition, with advances in the recognition of nontuberculous mycobacterium (NTM), prior studies have addressed the difficulty in distinguishing TB from NTM by using either clinical symptoms or imaging. ${ }^{3,4}$ The isolation prevalence of NTM has also increased gradually, further complicating the problem. ${ }^{5}$ Sputum AFB staining is one of the most readily accessible tools for evaluating patients suspected of having pulmonary TB; however, it is not specific for pulmonary TB. ${ }^{4,6}$ The aim of this retrospective case control study was to measure the impact of the misdiagnosis of pulmonary TB and to investigate which patients are at risk of receiving inappropriate treatment.

\section{Patients and methods}

\section{Patients}

From January 2008 to July 2011, we received 10,566 sputum specimens from 4427 patients at the laboratory of National Yang-Ming University Hospital (a 512-bed regional teaching hospital in Yilan, Taiwan) and the Far Eastern Memorial Hospital (a 1050-bed tertiary medical center in Taipei, Taiwan). Of these sputum specimens, 537 from 255 patients were AFB-stain-positive. Of these 255 patients, 15 were lost to follow-up during treatment and were excluded from the study.
Only 143 of the AFB stain-positive patients had proven pulmonary TB. The remaining 97 patients without pulmonary TB were enrolled for further analysis (Figure 1) and were divided into two groups according to whether or not they received anti-TB treatment. Of the 97 patients, 25 (25.8\%) were prescribed anti-TB drugs, mostly a combination of isoniazid, rifampicin, ethambutol, and pyrazinamide. The other $72(74.2 \%)$ patients were not initially diagnosed with pulmonary TB and were classified as the control group. The patients were followed up for 1 year. The study was approved by the Institutional Review Boards of National Yang-Ming University Hospital and Far Eastern Memorial Hospital.

\section{Diagnosis of pulmonary TB}

All of the sputum specimens were treated with Ziehl-Neelsen staining. Positive AFB stains were quantified as $1+$ to $4+(1+$, 1-9 AFB/100 fields: 2+, 1-9 AFB/10 fields: 3+, 1-9 AFB/ field: $4+,>9 \mathrm{AFB} /$ field $).{ }^{6} \mathrm{~TB}$ polymerase chain reaction $(\mathrm{PCR})$ was performed with in-house IS6110-based PCR assays. ${ }^{7}$ Mycobacterial cultures were performed using LöwensteinJensen medium. ${ }^{8}$ We retrospectively reviewed the medical records of all 240 patients with positive sputum AFB stains and analyzed the clinical and radiographic features. All chest computerized tomography and X-ray plain films were reviewed

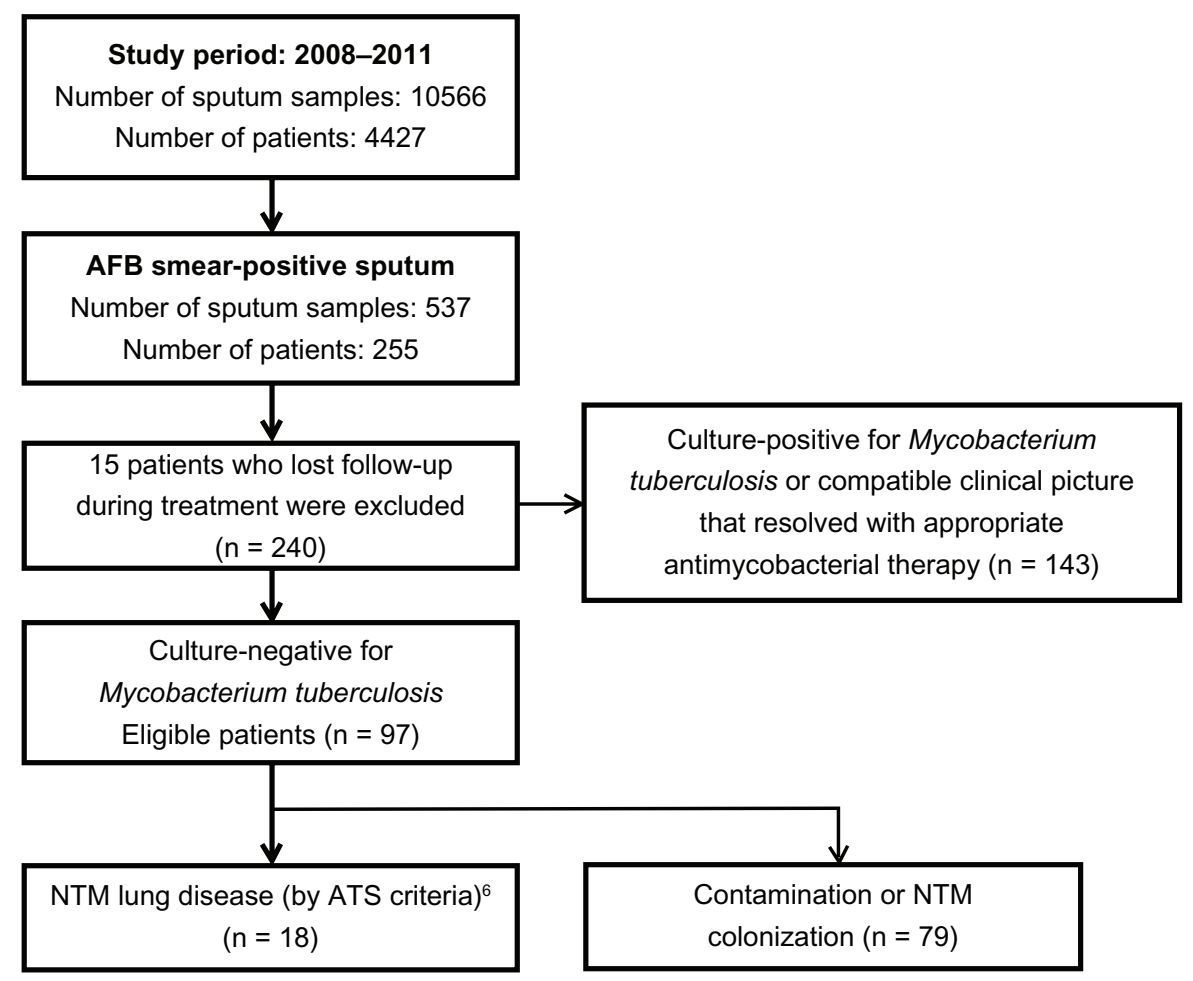

Figure I Flow diagram of study design.

Abbreviations: AFB, acid-fast bacilli; ATS, American Thoracic Society; NTM, nontuberculosis mycobacteria. 
by a radiologist (Yuan MK) and a pulmonologist (Chang CY), who were blinded to the clinical and epidemiological data. The diagnosis of pulmonary TB was made based on the American Thoracic Society pulmonary TB guidelines ${ }^{6}$ and was confirmed by our chest specialists. Patients were diagnosed with pulmonary TB if M. tuberculosis was isolated from their sputum or tissue specimens. For culture-negative patients with positive sputum AFB stains, if no other diagnosis was established and there was a radiographic or clinical improvement after 2 months of empirical anti-TB treatment, a diagnosis of pulmonary TB was made. ${ }^{9}$ The nucleic acid amplification test was not available in our laboratory and was not utilized in this study.

\section{Statistical analysis}

All statistical analyses were conducted using the software package SPSS 20.0 for Windows ${ }^{\circledR}$ (SPSS Inc., Chicago, IL, USA). Data are presented as frequencies for categorical variables, and by mean \pm standard deviation for numerical variables. Categorical variables were compared using a chi-square test or Fisher's exact test, and continuous variables were compared using an independent unpaired $t$-test. Univariate analysis was performed to evaluate the risk factors for inappropriate anti-TB treatment. Multivariate analysis was conducted using a logistic regression model to determine the independent predictive factors for inappropriate anti-TB treatment. $P$-values less than 0.05 were considered significant.

\section{Results}

\section{Demographic data}

From January 2008 to July 2011, 97 patients with positive sputum AFB smears who did not have pulmonary TB were enrolled. Their demographic data are summarized in Table 1. The mean age of the patients at enrollment was $69.3 \pm 16.2$ years, and $56.7 \%$ of the study population were male. The most common comorbidities were chronic obstructive pulmonary disease (19.6\%) and old pulmonary TB (18.6\%). Of the 97 patients with positive sputum AFB stains but no pulmonary TB, $18.5 \%(n=18)$ were NTM lung disease and $32 \%(n=31)$ were NTM colonization. The specimens from the remaining 48 patients yielded no pathogens. The most frequently isolated NTM was $M$. avium complex. None of the patients enrolled in this study had concomitant human immunodeficiency virus (HIV) infection.

\section{Adverse effect of inappropriate anti-TB treatment}

In the 97 patients with positive sputum AFB stains without pulmonary TB, 25 (25.8\%) were prescribed anti-TB treatment.
Table I Demographic data of patients

\begin{tabular}{ll}
\hline Age (years), mean \pm SD & $69.3 \pm 16.193$ \\
Range & $34-91$ \\
Male sex & $55(56.7)$ \\
Ever smoker & $21(21.6)$ \\
Comorbidities & \\
Diabetes mellitus & $12(12.4)$ \\
Old TB history & $18(18.6)$ \\
Autoimmune disease & $1(1)$ \\
COPD & $19(19.6)$ \\
Chronic kidney disease & $3(3.1)$ \\
Malignancy & $2(2.1)$ \\
Liver cirrhosis & $2(2.1)$ \\
Presenting symptom & \\
Chronic cough & $69(71.1)$ \\
Sputum production & $54(55.7)$ \\
Fever & $21(21.6)$ \\
Hemoptysis & $6(6.2)$ \\
Chest pain & $2(2.1)$ \\
AFB of sputum & \\
I+ & $76(78.4)$ \\
$2+$ & $11(11.3)$ \\
$3+$ & $8(8.2)$ \\
$4+$ & $2(2.1)$ \\
$2+$ to 4+ & $21(21.6)$ \\
Positive PCR & $12(12.4)$ \\
NTM lung disease & $18(18.6)$ \\
Received anti-TB treatment & $25(25.8)$ \\
\hline
\end{tabular}

Note: Data are $n(\%)$ unless specified otherwise.

Abbreviations: AFB, acid-fast bacilli; COPD, chronic obstructive pulmonary disease; NTM, non-TB mycobacteria; PCR, polymerase chain reaction; SD, standard deviation; $\mathrm{TB}$, tuberculosis.

In these 25 patients, eleven (44\%) patients developed adverse effects. The most common adverse effects were hepatitis and skin rash ( $\mathrm{n}=8$ and $\mathrm{n}=5$, respectively). Two patients suffered from gastrointestinal discomfort induced by anti-TB drugs. One patient developed gout, and one patient had visual impairment. None of the adverse effects in our patients were lethal and most were manageable after discontinuation of the offending agents.

\section{Comparison of clinical characteristics}

No statistically significant differences were found between the two groups with regards to age, sex, or the presence of underlying diseases such as chronic obstructive pulmonary disease and old TB. The presenting symptoms of both groups were similar; however, significantly more patients receiving inappropriate anti-TB treatment developed chronic cough (92.0\% versus $63.9 \%, P=0.008$ ) (Table 2 ). In addition, compared to the control group, patients with inappropriate anti-TB treatment had more acid-fast bacilli on sputum Ziehl-Neelsen staining ( $>10 / 100$ fields, grading $2+$ to $4+$; $36.0 \%$ versus $16.7 \%, P=0.043)$. 
Table 2 Comparison of clinical characteristics

\begin{tabular}{|c|c|c|c|}
\hline & $\begin{array}{l}\text { Treated } \\
\text { appropriately } \\
(n=72)\end{array}$ & $\begin{array}{l}\text { Treated } \\
\text { inappropriately } \\
(n=25)\end{array}$ & $P$-value \\
\hline \multicolumn{4}{|l|}{ Demographic data } \\
\hline Age (years), mean $\pm \mathrm{SD}$ & $70.58 \pm 15.738$ & $65.68 \pm 17.25$ & 0.194 \\
\hline Male sex & $4 \mid(56.9)$ & $14(56)$ & 0.935 \\
\hline Smoker & $16(22.2)$ & $5(20)$ & 0.816 \\
\hline Diabetes mellitus & $10(13.9)$ & $2(8)$ & 0.725 \\
\hline TB history & $12(16.7)$ & $6(24)$ & 0.551 \\
\hline Autoimmune disease & I (I.4) & $0(0)$ & 1 \\
\hline COPD & $17(23.6)$ & $2(8)$ & 0.142 \\
\hline Chronic kidney disease & $3(4.2)$ & $0(0)$ & 0.567 \\
\hline Malignancy & $2(2.8)$ & $0(0)$ & 1 \\
\hline Liver cirrhosis & $2(2.8)$ & $0(0)$ & 1 \\
\hline \multicolumn{4}{|l|}{ Presenting symptom } \\
\hline Chronic cough & $46(63.9)$ & $23(92)$ & 0.008 \\
\hline Sputum & $38(52.8)$ & $16(64)$ & 0.33 \\
\hline Fever & $15(20.8)$ & $6(24)$ & 0.74 \\
\hline Hemoptysis & $6(8.3)$ & $0(0)$ & 0.334 \\
\hline Chest pain & $0(0)$ & $2(8)$ & 0.064 \\
\hline \multicolumn{4}{|l|}{ AFB of sputum } \\
\hline $2+$ to $4+$ & $12(16.7)$ & $9(36)$ & 0.043 \\
\hline Positive PCR & $7(9.7)$ & $5(20)$ & 0.287 \\
\hline
\end{tabular}

Note: Data are $\mathrm{n}(\%)$ unless specified otherwise.

Abbreviations: AFB, acid-fast bacilli; COPD, chronic obstructive pulmonary disease; PCR, polymerase chain reaction; SD, standard deviation; TB, tuberculosis.

\section{Comparison of radiographic characteristics}

There were no significant differences in the radiographic analysis between the two groups (Table 3).

\section{Multivariate analysis}

Multivariate analysis showed that chronic cough was a significant predictor for inappropriate anti-TB treatment in the patients with positive sputum AFB stains who did not have true pulmonary TB $(P=0.026)$. Although the patients with heavier sputum AFB stains $(\geq 2+)$ had an increased risk of receiving inappropriate anti-TB treatment on univariate analysis, the trend was not statistically significant on multivariate analysis $(P=0.095)$.

\section{Discussion}

Although administering anti-TB treatment empirically for patients likely to have pulmonary TB in endemic areas is justified, $40.4 \%$ of patients with positive sputum AFB stains ( $n=97$ ) did not have pulmonary TB in the current study. In addition, in those who had positive sputum AFB stains without pulmonary TB, 25.8\% $(n=25)$ were prescribed anti-TB treatment and $44 \%$ of these $(n=11)$ developed various adverse effects (hepatitis: $32 \%$, skin rash: $20 \%$ ). None of
Table 3 Comparison of radiographic characteristics

\begin{tabular}{|c|c|c|c|}
\hline & $\begin{array}{l}\text { Treated } \\
\text { appropriately } \\
(\mathbf{n = 7 2}) \\
\end{array}$ & $\begin{array}{l}\text { Treated } \\
\text { inappropriately } \\
(n=25)\end{array}$ & $P$-value \\
\hline \multicolumn{4}{|c|}{ Chest plain film $(n=97)$} \\
\hline Affected lung & & & $0.84 I$ \\
\hline Unilateral & $17(23.6)$ & $5(20)$ & \\
\hline Bilateral & $44(61.1)$ & $17(68)$ & \\
\hline Pleural effusion & & & 0.536 \\
\hline Unilateral & $10(13.9)$ & $4(16)$ & \\
\hline Bilateral & $5(6.9)$ & $0(0)$ & \\
\hline Pleural lesion & $12(16.7)$ & $4(16)$ & I \\
\hline Fibrothorax & $3(4.2)$ & I (4) & I \\
\hline Cavitation & $0(0)$ & I (4) & 0.258 \\
\hline Consolidation & $16(22.2)$ & $3(12)$ & 0.383 \\
\hline $\begin{array}{l}\text { Nodular or } \\
\text { reticulonodular }\end{array}$ & $48(66.7)$ & $19(76)$ & 0.384 \\
\hline Honeycomb & $4(5.6)$ & $2(8)$ & 0.646 \\
\hline Atelectasis & $16(22.2)$ & $5(20)$ & 1 \\
\hline
\end{tabular}

Note: Data are $\mathrm{n}(\%)$ unless specified otherwise.

the adverse effects in our patients were lethal; however, they did cause unnecessary harm.

Of the 97 patients with positive sputum AFB stains but no pulmonary TB, $18.5 \%(\mathrm{n}=18)$ were later diagnosed with NTM lung disease and $32 \%(n=31)$ were afflicted with NTM colonization. The most frequently isolated NTM was M. avium complex. In total, $61.1 \%$ of these 18 patients $(n=11)$ initially received inappropriate anti-TB treatment. This result is consistent with prior reports of an increased culture prevalence of NTM and the difficulty in distinguishing pulmonary TB and NTM based on symptoms, signs, and radiological features. ${ }^{4}$ The performance of LöwensteinJensen medium in our study was similar to that reported by Muyoyeta et al. ${ }^{10}$ In their report, only $<50 \%$ of smear positive sputum specimens yielded TB with LöwensteinJensen medium and nearly $50 \%$ had a negative result. Very few NTM isolates were recovered $(<1 \%)$. In the current study, however, we isolated much more NTM than in the aforementioned study: in our 48 smear-positive but culturenegative patients, it is unlikely that they suffered from undiagnosed pulmonary TB, since they were followed up for more than 1 year to ensure that culture-negative TB could be recognized clinically; therefore, it is possible that the remaining culture negative cases were either contaminated or had unidentified NTM colonization.

In our study, physicians tended to administer inappropriate and unnecessary empiric anti-TB treatment for patients with AFB smear-positive sputum who had chronic cough or heavier AFB staining $(P=0.008$ and $P=0.043$, respectively). 
For patients with a chronic cough or heavier sputum AFB staining, this result is quite reasonable, as pulmonary TB is mainly transmitted via airborne routes. ${ }^{9}$ In addition, more AFB found in the sputum may reflect more pathogens in the lungs. Both of these factors imply a high risk of disease transmission, ${ }^{11}$ and hence it is understandable that doctors would treat these patients empirically. According to our study, however, many patients with heavy AFB staining did not have true pulmonary TB. In addition, a positive AFB stain can be caused by various conditions other than pulmonary TB, such as Nocardia and NTM. ${ }^{12}$ If the importance and implication of heavy AFB staining are overestimated, unnecessary anti-TB treatment may be administered. This finding is consistent with the report of Pokam and Asuquo ${ }^{12}$ in sub-Saharan Africa, where laboratory culture facilities are lacking and treatment is solely based on sputum AFB stains.

We also analyzed the radiographic features of the patients to see if there were any specific radiographic patterns associated with misdiagnosis and administration of unnecessary anti-TB treatment. However, there were no significant differences between the two study groups.

Despite the recent advances in the BACTEC MGIT (Mycobacteria Growth Indicator Tube) 960 System (BD, Franklin Lakes, NJ, USA), which greatly shortens the time required for culturing mycobacteria from 6-8 weeks to 2-4 weeks, the time required to confirm a diagnosis is still significant. ${ }^{13,14}$ Before the results of mycobacterial cultures are obtained, patients without true pulmonary TB may receive inappropriate and unnecessary anti-TB treatment for weeks. TB PCR techniques have improved this situation; however, they are relatively costly and their lower availability limits their use in some endemic areas. In countries with more limited resources, it is not possible to incorporate PCR into routine clinical use, and the diagnosis is still based largely on clinical symptoms and sputum AFB stains. ${ }^{4,12}$ With regards to the overall performance of the in-house IS6110-based PCR we used, the sensitivity to detect M.tuberculosis in patients with AFB smear-positive sputum in this study was $58.9 \%$, with a specificity of $87.6 \%$ and a false positive rate of $12.37 \%$.

There are some limitations to this study. For instance, we enrolled only 97 patients in the study and thus the sample size is relatively small. Second, the prevalence and management of NTM are different in patients with HIV infection. None of the patients enrolled in this study had concomitant HIV infection, and it is therefore not possible to extrapolate the results of the study to patients with HIV infections. The purpose of this study was to investigate the risk factors for unnecessary antiTB treatment in patients with AFB smear-positive sputum without pulmonary TB; we did not analyze the patients with pulmonary TB and use them as the control group. Therefore, it is not known whether there were other factors causing the patients without pulmonary TB to mimic TB patients clinically, thereby misleading clinicians.

Our findings in this study address the issue that clinical symptoms combined with AFB positive sputum smear do not allow for accurate differentiation between TB and NTM infection or NTM environmental contamination. In endemic areas, immediate and empirical treatment based on the result of AFB sputum smear has an important impact on disease transmission control. However, the administration of unnecessary empiric anti-TB treatment is not uncommon in real world practice and physicians should be careful while rendering a decision to treat these patients. Withholding anti-TB treatment and waiting for further confirmation by either PCR or mycobacterial cultures may reduce the administration of unnecessary treatment. However, postponing treatment may have a negative impact on public health and disease transmission control. More studies are warranted to elucidate this issue.

\section{Conclusion}

Initiating anti-TB treatment solely based on clinical features is not reliable and may lead to inappropriate treatment. The main risks factors related to unnecessary empiric anti-TB treatment in this study were chronic cough and heavy sputum AFB staining $(\geq 2+)$.

\section{Disclosure}

The authors report no conflicts of interest in this work.

\section{References}

1. Donald PR, van Helden PD. The global burden of tuberculosis combating drug resistance in difficult times. N Engl J Med. 2009;360(23): 2393-2395.

2. Blumberg HM, Burman WJ, Chaisson RE, et al; American Thoracic Society, Centers for Disease Control and Prevention and the Infectious Diseases Society. American Thoracic Society/Centers for Disease Control and Prevention/Infectious Diseases Society of America: treatment of tuberculosis. Am J Respir Crit Care Med. 2003;167(4):603-662.

3. Al Jarad N, Demertzis P, Jones DJ, et al. Comparison of characteristics of patients and treatment outcome for pulmonary non-tuberculous mycobacterial infection and pulmonary tuberculosis. Thorax. 1996;51(2): 137-139.

4. Maiga M, Siddiqui S, Diallo S, et al. Failure to recognize nontuberculous mycobacteria leads to misdiagnosis of chronic pulmonary tuberculosis PLoS One. 2012;7(5):e36902.

5. Marras TK, Chedore P, Ying AM, Jamieson F. Isolation prevalence of pulmonary non-tuberculous mycobacteria in Ontario, 1997-2003. Thorax. 2007;62(8):661-666. 
6. [No authors listed]. Diagnostic Standards and Classification of Tuberculosis in Adults and Children. This official statement of the American Thoracic Society and the Centers for Disease Control and Prevention was adopted by the ATS Board of Directors, Jul 1999. This statement was endorsed by the Council of the Infectious Disease Society of America, September 1999. Am J Respir Crit Care Med. 2000;161(4 Pt 1): 1376-1395.

7. Sun JR, Lee SY, Perng CL, Lu JJ. Detecting Mycobacterium tuberculosis in Bactec MGIT 960 cultures by inhouse IS6110-based PCR assay in routine clinical practice. J Formos Med Assoc. 2009;108(2):119-125.

8. Tarshis MS, Kinsella PC, Parker MV. Blood media for the cultivation of Mycobacterium tuberculosis. VII. Comparison of blood agar-penicillin and Lowenstein-Jensen media under routine diagnostic conditions. J Bacteriol. 1953;66(4):448-452.

9. American Thoracic Society, Centers for Disease Control and Prevention, Infectious Diseases Society of America. American Thoracic Society/ Centers for Disease Control and Prevention/Infectious Diseases Society of America: controlling tuberculosis in the United States. Am J Respir Crit Care Med. 2005;172(9):1169-1227.
10. Muyoyeta M, Schaap JA, De Haas P, et al. Comparison of four culture systems for Mycobacterium tuberculosis in the Zambian National Reference Laboratory. Int J Tuberc Lung Dis. 2009;13(4): 460-465.

11. Lohmann EM, Koster BF, le Cessie S, Kamst-van Agterveld MP, van Soolingen D, Arend SM. Grading of a positive sputum smear and the risk of Mycobacterium tuberculosis transmission. Int J Tuberc Lung Dis. 2012;16(11):1477-1184.

12. Pokam BT, Asuquo AE. Acid-fast bacilli other than mycobacteria in tuberculosis patients receiving directly observed therapy short course in cross river state, Nigeria. Tuberc Res Treat. 2012;2012:301056.

13. Somoskövi A, Ködmön C, Lantos A, et al. Comparison of recoveries of mycobacterium tuberculosis using the automated BACTEC MGIT 960 system, the BACTEC 460 TB system, and Löwenstein-Jensen medium. J Clin Microbiol. 2000;38(6):2395-2397.

14. Morgan MA, Horstmeier CD, DeYoung DR, Roberts GD. Comparison of a radiometric method (BACTEC) and conventional culture media for recovery of mycobacteria from smear-negative specimens. J Clin Microbiol. 1983;18(2):384-388.
Drug Design, Development and Therapy

\section{Publish your work in this journal}

Drug Design, Development and Therapy is an international, peerreviewed open-access journal that spans the spectrum of drug design and development through to clinical applications. Clinical outcomes, patient safety, and programs for the development and effective, safe, and sustained use of medicines are a feature of the journal, which

\section{Dovepress}

has also been accepted for indexing on PubMed Central. The manuscript management system is completely online and includes a very quick and fair peer-review system, which is all easy to use. Visit $\mathrm{http}: / /$ www.dovepress.com/testimonials.php to read real quotes from published authors.

Submit your manuscript here: http://www.dovepress.com/drug-design-development-and-therapy-journal 